\title{
Factors influencing physical activity in adults with cystic fibrosis
}

\author{
Nicola Hurley ${ }^{1 *}$, Niall M. Moyna ${ }^{1}$, Bróna Kehoe ${ }^{2}$, Noel McCaffrey ${ }^{3}$, Karen Redmond ${ }^{4}$ and Sarah J. Hardcastle ${ }^{1,5}$
}

\begin{abstract}
Background: Physical activity (PA) is a well-documented and accepted adjunct therapy for the maintenance and improvement of long-term health in cystic fibrosis (CF). Although the benefits of PA for CF populations are well-established, adherence to PA programmes within this population remains low. This study aimed to investigate the factors that influence engagement in physical activity, and to explore exercise preferences, among adults with cystic fibrosis (CF).

Methods: Semi-structured telephone interviews were conducted. Participants were twenty-one adults (mean age 35 years, $\mathrm{SD} \pm 8$ ) with an established diagnosis of $C F$, living in Ireland. Interview scripts were digitally recorded and transcribed verbatim. Thematic analysis was used to analyse the data.
\end{abstract}

Results: Four main themes emerged: barriers, motives, value of exercise-related outcomes, and exercise preferences. The main barriers included: low energy levels, time, the weather, and exercise-related confidence. Enjoyment and perceived competence underpinned autonomous motivation. Participants who self-identified as being regularly active valued personally identified exercise-related outcomes such as, accomplishment and affect regulation. Participants indicated a preference for home-based physical activity programs compared to gym- or facility-based programs.

Conclusion: Interventions aimed at promoting physical activity among adults with CF should involve programs that foster autonomous motivation, enjoyable activities, personally identified outcomes, competence and that can be conducted from the home environment.

Clinical implications: To increase physical activity participation among adults with $C$, interventions that can be conducted from the home environment, that pay attention to the patients' personally-valued exercise outcomes may be required.

Keywords: Cystic fibrosis, Exercise, Physical activity, Psychology, Motivation, Psychosocial, Behaviour change

\section{Introduction}

Cystic fibrosis (CF) is a progressive, multi-system, inherited condition affecting approximately 1400 people in Ireland, and more than 70,000 worldwide $[1,2]$. Today, with advancements in early diagnosis and the development of highly effective $\mathrm{CF}$ transmembrane regulator modulator therapies, people with CF are living longer [3, 4]. As such,

\footnotetext{
*Correspondence: nicola.hurley5@mail.dcu.ie

${ }^{1}$ School of Health and Human Performance, Faculty of Science

and Health, Dublin City University, Dublin 9, Ireland

Full list of author information is available at the end of the article
}

increased emphasis is being placed on improving lifestyle-related behaviours in order to enhance long-term health in adults with CF [5].

Aerobic fitness has been shown to be an important predictor of survival in individuals with $\mathrm{CF}$ [6]. Those who have a higher aerobic capacity $\left(\mathrm{VO}_{2} \geq 82 \%\right.$ predicted $\mathrm{VO}_{2} \max$ ) have an increased survival rate of $83 \%$ at 8 -years, compared to $51 \%$ and $28 \%$ survival with moderate $\left(59-81 \%\right.$ predicted $\left.\mathrm{VO}_{2} \max \right)$ and low fitness $(\leq 58 \%)$, respectively [7]. In a recent systematic review and metaanalysis including six studies (2019), Vendrusculo et al. 
[8] have shown that lower $\mathrm{VO}_{2}$ max levels are associated with an increase of 4.9 in the risk of mortality in People with Cystic Fibrosis (PWCF). Evidence suggests that there is a positive relation between increased physical activity (PA) at moderate intensity and improvements in aerobic capacity, independent of improvements in muscle strength and pulmonary function $[9,10]$. Regular participation in physical activity (PA); performing 150-min/ wk, and preferably 300-min/wk of moderate-to-vigorous PA [1] improves a myriad of outcomes in CF, such as exercise tolerance [11], airway clearance [12] energy levels [13] and quality of life [14]. Sustained PA also has the potential to slow the annual rate of decline in pulmonary function [15]. Despite the established benefits of PA for individuals with $\mathrm{CF}$, adherence to PA programs within this population can be poor $[15,16]$. In order to develop the optimal PA program for individuals with CF, a better understanding of patient attitudes towards PA is needed to elicit sustainable lifestyle behaviours [5].

Few studies have investigated the factors influencing exercise participation in adults with CF [5, 16-19]. The most commonly reported motivators for sustained PA participation included enjoyment [17], motivation [17], improving general and/or lung-health [16], and feeling healthy [16]. Feelings of breathlessness [17], fatigue [18], lack of good health [5], reduced energy [5], and embarrassment when exercising in public [18] were among the most common barriers reported. Myers et al. [19] suggested severity of CF lung disease and being female were associated with more significant and rapid decline in lung function, as factors that may contribute to poorer longterm adherence to PA. Lack of motivation and time were also reported as barriers to exercise [5, 17].

Several models have been developed to provide a more thorough understanding of the relation between PA and behaviour change. The theory of planned behaviour focuses on beliefs and attitudes and social norms [20], Bandura's social cognitive theory [21] focuses primarily on self-efficacy and outcome expectancies, and the selfdetermination theory [22] focuses on the quality of motivation and the importance of satisfying key psychological needs such as autonomy and competence-relatedness. To date, there is no optimal model to explain PA and behaviour change, and qualitative research has therefore been helpful, by not taking a deductive approach and approaching the topic with a broad perspective to unravel which dimensions appear to be most important [23]. The current study is not aligned to any particular theoretical framework as it is inductive in nature, and will explore the dimensions that influence PA behaviour change in a CF population, and therefore provide an insight into which behaviour change models may be most suitable when designing interventions. Given previous research findings and the aforementioned theories, it is expected that low self-competence and motivation may be theoretical components of emerging barriers to exercise in the CF cohort [23].

The purpose of this study was to explore the attitudes towards, and dimensions influencing, PA participation in Irish adults with CF. Such knowledge concerning the dimensions influencing PA and attitudes towards PA would be beneficial in the development of appropriate intervention strategies to promote PA in practice.

\section{Materials and methods}

The current study conformed to suggested guidelines by Clark in appropriateness of method, transparency of procedures, and soundness of approach [24].

\section{Participant recruitment}

Convenience sampling was used to recruit adults with an established diagnosis of CF (confirmatory diagnostic sweat chloride test). An email including a patient information leaflet was circulated by CF Ireland, the national CF charity, to all adults with $\mathrm{CF}$ on the registry in Ireland $(n=861)$. Following an expression of interest, participants were screened for eligibility. Participants were eligible for inclusion in the study if they were $\geq 18$ years of age and living in Ireland. Written informed consent was obtained in accordance with the Dublin City University (DCU) Research Ethics Committee (DCUREC/2018/141).

\section{Data collection}

Semi-structured telephone interviews lasting up to 30-min were conducted. An interview guide was developed based on previously published work (see Additional file 1) [25], regarding the factors that may influence PA in adults with CF. Interviews were digitally recorded and transcribed verbatim. Interviews were conducted in the Autumn of 2019 (September-October).

\section{Data analysis}

Data were analyzed inductively using thematic analysis, which involved several steps [26]. The first step involved active and repeated reading of the transcripts to gain an insight into the data, and to explore initial codes. The second step involved systematically (i.e., line by line) coding features of interest within the data (data extracts) that are related to the research question. The third step involved exploring whether codes may be collated to form overarching themes. This step, which re-focuses the analysis at the broader level of themes, rather than codes, involves sorting the different codes into potential themes, and collating all the relevant coded data extracts within the identified themes. A theme captures something important 
about the data in relation to the research question and represents some degree of patterned response within the data set. Confidence of the existence of a theme is established when a number of instances of the theme are identified across the data set or across the sample [26]. The fourth step involved reviewing and refining the potential themes. This phase involved checking whether there was sufficient data to support the existence of a theme or whether there was overlap such that themes might collapse into each other (e.g., two apparently separate themes might form one theme). In step four, the researchers' checked for coherence between the data and the themes and checked for identifiable distinctions between themes. The final step involved the refining and defining of themes. Once a satisfactory thematic map of the data was achieved, themes were further refined and defined to be presented for analysis and cross-checked the data extracts within them. During this step, theme and sub-themes were reviewed to ensure that they matched the data and that each theme is coherent and internally consistent (i.e., not too much overlap between themes). As part of the refinement, decisions were also made concerning hierarchy and the presence of sub-themes that fit into a broader overarching theme. Sub-themes are used for giving structure to a particularly large and complex theme, and also for demonstrating the hierarchy of meaning within the data.

A second researcher experienced in qualitative data analysis served as a second reader of the transcripts and offered further insight with respect to emergent themes to broaden data interpretation and assist with coding, reviewing and refining of themes. Data saturation was determined following analysis and occurred at the point when no new information was gained to develop or broaden themes [27]. Credibility and rigor are demonstrated in the analysis in the following ways; (1) using data saturation to determine sample size, (2) describing in detail the steps taken in conducting the analysis, (3) involving two researchers in the process of analysis thereby broadening the number of interpretations and (4) providing 'thick description' via the use of extensive and direct quotations so that the reader can evaluate the interpretation (i.e., the fit between data extracts and the theme labels) [28].

During the thematic analysis process, the researcher ensured that each transcript was labelled according to self-reported activity status, using 'low-active' defined as not meeting the current consensus CF PA guidelines, 'moderately active' defined as meeting, or likely to be meeting, the guidelines, or 'highly active' defined as exceeding the minimum PA guidelines for PWCF, on the front page to identify potential differences between responses according to activity status.

\section{Results}

Twenty-one adults (57\% males) with an established diagnosis of CF participated in the study. The mean \pm SD age of subjects was $35 \pm 8$ years, with $16 \%$ of participants having undergone lung transplantation. Participants' pseudonyms, characteristics and demographics are outlined in Tables 1 and 2. Table 3 displays participant characteristics compared to the Irish CF population, and indicates that the sample in the present study is broadly representative of the Irish CF population in relation to gender, $\mathrm{BMI}$ and presence of at least one copy of the $\Delta \mathrm{f} 508$ gene. However, participants in the present study were older (median 34 years vs 21 years), with higher lung function ( $89 \%$ vs $82.3 \%$ ), and a greater proportion had received a lung transplant ( $14 \%$ vs $5 \%$ ).

Data analysis identified four key themes relating to factors that influence PA engagement in adults with CF: (1) barriers underpinned by four subthemes including energy levels, time, weather and exercise-related confidence; (2) motivation with sub-themes of enjoyment and perceived competence; (3) valued outcomes, underpinned by subthemes of accomplishment and affect regulation; and (4) preferences (refer to thematic map in Fig. 1). Each quote is followed by a pseudonym and the individual's age. To further contextualize the data, self-reported PA and additional quotes are provided in Tables 3 and 4 . In

Table 1 Participant pseudonyms and individual characteristics

\begin{tabular}{|c|c|c|c|c|c|}
\hline ID code & Pseudonym & Gender & Age & Self-reported PA & $\begin{array}{l}\text { transplant } \\
\text { status }\end{array}$ \\
\hline 1 & Paul & Male & 30 & High & No \\
\hline 2 & Mary & Female & 30 & Moderate & No \\
\hline 3 & Jenny & Female & 44 & Low & Yes \\
\hline 4 & William & Female & 34 & Low & Yes \\
\hline 5 & Richard & Male & 49 & Low & No \\
\hline 6 & Matthew & Male & 28 & High & No \\
\hline 7 & Shane & Male & 33 & High & No \\
\hline 8 & Robert & Male & 41 & High & No \\
\hline 9 & Emily & Female & 50 & High & No \\
\hline 10 & Dorothy & Female & 29 & Moderate & No \\
\hline 11 & Carol & Female & 36 & High & No \\
\hline 12 & Kevin & Male & 33 & High & No \\
\hline 13 & Amy & Female & 32 & Low & Yes \\
\hline 14 & Eric & Male & 50 & Low & No \\
\hline 15 & Brenda & Female & 35 & Moderate & No \\
\hline 16 & Frank & Male & 26 & High & No \\
\hline 17 & Peter & Male & 19 & Low & No \\
\hline 18 & Keith & Male & 38 & High & No \\
\hline 19 & Hannah & Female & 36 & Low & No \\
\hline 20 & John & Male & 31 & High & No \\
\hline 21 & Jason & Male & 35 & Low & No \\
\hline
\end{tabular}


Table 2 Participant demographics

\begin{tabular}{ll}
\hline $\mathrm{FEV}_{1}(\%$ predicted $)$ & $84.1 \pm 19.7$ \\
$\mathrm{BMI}\left(\mathrm{kg} \cdot \mathrm{m}^{2}\right)$ & $24.1 \pm 2.4$ \\
Diabetes Status & CFRD $(\mathrm{n}=2)$ \\
Pancreatic Status & Pancreatic Insufficiency $(\mathrm{n}=5)$ \\
Co-existing Conditions & Neurocardiogenic syncope; Osteopenia; Asthma; Nasal \\
& Polyps; CF Liver Disease; Gastro-intestinal Reflux; CF-related \\
Homozygous $\triangle F 508$ & Arthritis \\
Heterozygous $\triangle F 508$ & 4 \\
Other CF Alleles & 3 \\
Location of CF Centre & G542x; R117H; E60x \\
\hline
\end{tabular}

Results are reported as mean $\pm S D$

Results for FEV 1 , BMI, CF Alleles, Diabetes and Pancreatic Status for 9/21 participant

Table 3 Study participant characteristics compared to the Irish CF population

\begin{tabular}{lll}
\hline & Study CF population & Irish CF population \\
\hline Age (Years) & Median: 34 & Median: 21 \\
Gender (Males: Females) & $42 \%: 58 \%$ & $42 \%: 58 \%$ \\
BMl (kg. $\mathrm{m}^{2}$ ) & Median: 23.4 & Median: 22.3 \\
FEV $_{1}(\%$ predicted) & 89 & 82.3 \\
Genotype (at least 1 copy of & $87.5 \%$ & $91.7 \%$ \\
$\quad \triangle$ f508) & $14 \%$ & $5 \%$ \\
\hline Transplant Status & & \\
\hline
\end{tabular}

Data for BMI, FEV 1 and Genotype are only available for 8/21 participants. Data regarding the Irish CF population was obtained from the CF Registry in Ireland Annual Report for 2019

accordance with Sandelowski (2000), pronouns will represent indeterminate quantities where 'many' implies approximately $75 \%$, 'several' implies approximately $50 \%$, and 'few' implies approximately $20 \%$ of the sample [29].

\section{Barriers}

Participants reported both internal and external barriers to PA participation. The most common internal barrier was low energy: "There are some days where I'm just really mentally drained...So, the idea then of having to get up on a bike can be a huge barrier because I just feel so exhausted" (Amy, 32); "I'll just be generally really tired...so that might get in the way of being motivated, I don't want to push myself (Dorothy, 29); "Energy would be a big one. Sometimes I've just enough energy to get up and do my nebs, and I don't really have the energy to do other things" (Hannah, 50). Participants also reported barriers pertaining to exercise-related confidence, such as self-consciousness: "In gyms and public when you're coughing, there's people looking at you to say what is wrong with you?" (Eric, 50), and concerns of capability; "The thing that turns me off PA is the fear of not being able to do [it], if that makes sense? Everyone in the class is jumping up and down on the step and I'm like oh my God, I can't keep up" (Hannah, 36).

External barriers to regular participation in PA included time: "I just generally don't think I get the time" (Mary, 30); "Essentially it's time. Time is the major one for me... prior to the kids...I was getting much more exercise, but time is the main thing" (Richard, 49); and the weather: "The weather here in Ireland is just...you could plan to go and have a round of golf, or play tennis at the weekend, and then it's raining all day so that stops that" (Matthew, 28); "If the weather is bad, my motivation is squat. I'm very much a fair-weather walker, you know?" (Jenny, 44).

\section{Motivation}

Motivation was a dominant theme identified throughout, with several participants displaying higher levels of motivation: "I'm always very self-motivated actually, I suppose from a young age I always kind of realised that..., it's me that's going to do it, nobody else can do it for me" (Amy, 32); "I'm very self-motivated, I don't need somebody shouting at me in a gym" (John, 31), compared to others who were primarily motivated by external drivers, such as guilt: "It's more guilt than anything I have to say. I go through stages of doing it but it's guilt more than anything else" (Eric, 50); and "It's hard to keep motivated because you know you have to do it" (Mary, 30). Enjoyment and perceptions of competence appeared to underpin PA motivation.

\section{Enjoyment}

Participants who expressed positive attitudes towards and enjoyment of PA: "It's always been a positive, I have been really active most of my life now" (Kevin, 33); "Yeah, very positive. I feel the more exercise I do, the better I 


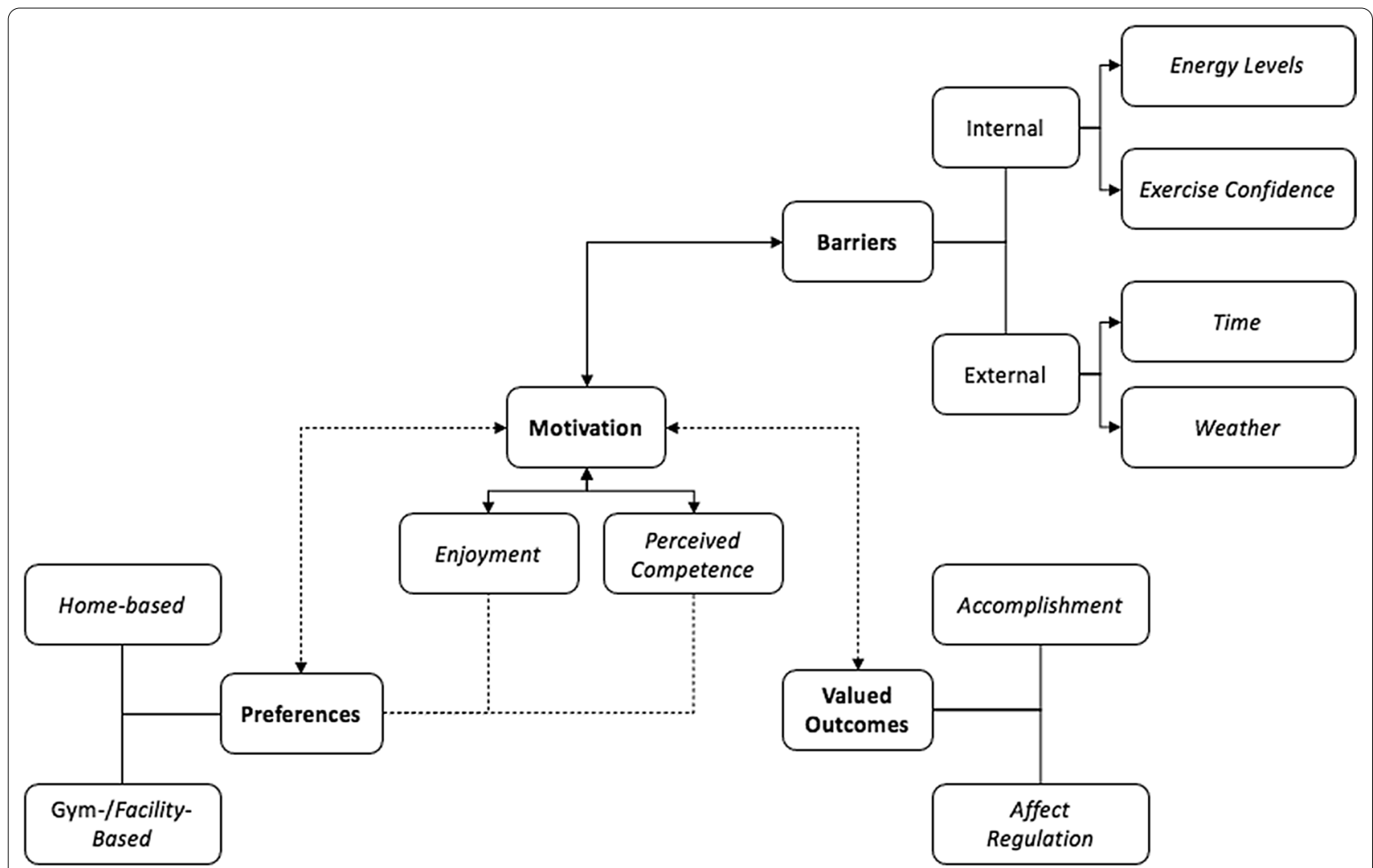

Fig. 1 Thematic Map Displaying Themes and Relationship between themes. The thematic map displays the four primary themes (motivation, barriers, valued outcomes and preferences), and sub-themes. The arrows provided reflect the relationship between and within themes. For example, an individual who values exercise outcomes (through feelings of accomplishment or through affect regulation) is more motivated to exercise. Enjoyment of exercise and perceptions of competence affect motivation for PA and also preferences

feel" (Richard, 49), seemed to engage in more regular PA: "I go to the gym five days a week; I do twenty-five minutes of cardio and then I do one particular muscle"' (Kevin, 33), and "I'm very active, I'd get up at 7 o'clock I go for a $3 \mathrm{~km}$ run...then in the afternoon I go to the gym for an hour weight session. And I do that for 2 days and then I have the third day off...then I go through all that again" (Robert, 41). Exercise enjoyment appeared to be a motivating factor for adherence: "I think the main thing is to do something you enjoy. Anytime I've been successful at kind of maintaining sport, it's just doing something I enjoy" (William, 34); "I suppose ...the main reason is, I enjoy it" (Matthew, 28).

In contrast, some participants reported a lack of enjoyment as a reason for their physical inactivity: "None of it. I'm going to be very honest with you, I do not enjoy PA at all" (Eric, 50); “To be honest, I don't particularly enjoy it!" (Mary, 30); "Mainly, I just don't like exercise" (Peter, 19), which was typically underpinned by poor engagement: "At the moment I would say my PA is pretty much zero... I haven't been doing much exercise" (Peter, 19), and/or negative past experiences with PA: "Very negative. For me, PA was just hard" (Jenny, 44); "I suppose negative. I wouldn't be going to the gym. I wouldn't be interested in any of that" (Eric, 50).

\section{Perceived competence}

Several participants reported high levels of perceived competence that appeared to underpin their exercise motivation: "You know, even though I have this illness, I never really felt like I did because it really didn't keep me from setting records on the leader board when I was younger...I was very fast" (Carol, 36): "But it's just the realization that $\mathrm{I}$ am as fit as most of them in the room and it's at that... and keeping that level up, it's keeping me on par with most of them in the room and for my own benefit as well" (Robert, 41); "I know I'm well able to do it" (Emily, 50).

Other participants did not identify as being the sporty or exercise type: "I've probably never been that person. I was never a sporty person" (Jenny, 44); “Exercise wouldn't be in my remit. I wouldn't be interested in any of that. I wouldn't be a gym bunny; I'd be far from it. As far away from it as you can imagine" (Eric, 50), and expressed low 
Table 4 Overview of themes and illustrative quotes

Theme

Low energy levels were dominant internal barriers to PA engagement

Time and the weather were dominant external barriers to PA engagement

Positive past PA experiences were typically associated with more regular PA participation, driven predominantly by enjoyment

Value of exercise-related outcomes: accomplishment and affect regulation
Illustrative quotes

"It's just because I know it will be tiring" (Jenny, 44)

"Just when I'm feeling unwell, lack of energy. Most days I don't be in the mood to go to the gym, just through fatigue and tiredness" (Robert, 41)

"Low energy, if I'm not feeling the best, I'd use that as an excuse" (Emily, 50)

"Some days I'm just absolutely wrecked, like I can be so tired. I just don't want to move, just don't want to go out, sometimes I don't even want to sweep the floor in the house, it's that type of...just comes out of nowhere" (Paul,30)

"Energy wise, yeah, I find my energy levels are even when I wake up in the morning are low" (Keith, 38)

"I have just started college and on the days where I'm in, I know that I don't have the energy to work out and a full day in college on the same morning" (Dorothy, 29)

"it's probably like time and weather" (Frank, 26)

"I suppose time is one thing, obviously having CF and working full time and family it's tough" (Keith, 38)

"Time is definitely one as well" (Hannah, 36)

"I work kind of in the evenings times 3 to 11 at night. So going to the gym I have to be up early to kind of go in and get to the gym early. And I have no problem with doing that, it's just at times you wake up and you feel so exhausted from work that it's just too difficult. Whereas if I had a schedule around 9 to 5, I'd go to the gym on the way home l'd do it more often" (Jason, 35)

"The weather is getting bad, so, going to be stuck in doors more, will be hard to get up there, the motivation might slack a bit" (Paul, 30)

"My motivation if the weather is [bad], my motivation is squat" (Jenny, 44)

"As I say that's weather depending" (Richard, 49)

"The Irish weather I find is a barrier for me to get to be physically active" (Matthew, 28)

"I don't walk in torrential rain and... the winter months" (Jenny, 44)

"Positive, from the age of about, when I was in school and stuff, and I was always playing sport, I was always playing football" (Robert, 41)

"Positive, I always try to keep active... I have always kind of done it throughout my life" (Emily, 50)

"So, as a kid, I wouldn't have noticed any difference from anyone else, I don't think. Very active" (Hannah 36)

"So, if I go for a run first thing in the morning it helps me clear all the mucus and stuff in my lungs. Then the afternoon I go to the gym for an hour weight session. And I do that for 2 days and then I have the third day off. And then I go through all that again." (Robert, 41)

"I usually try and go to the gym 3 mornings a week and that would include 2 spinning classes and a hit class, high intensity class. So they would each last approximately 30 min each but they would be quite physical." (Emily, 50)

"Yes, so at the minute I am exercising probably 5 or 6 days a week...I would run on the treadmill for about 10 or 15 min depending on how I'm feeling between 6 to 7.5 miles, that's like speed wise. So, then l'd do some weights I do the sit ups" (Dorothy, 29)

"I've always really enjoyed the exercise." (William, 34)

"I do enjoy the physical aspect of it, I enjoy putting my music on and just running the treadmill" (Dorothy, 29)

"it's fun and it's enjoyable" (Carol, 36)

"I enjoy kind of the buzz you get... the way it clears your head...you kind of feel better afterwards" (William, 34) 
Table 4 (continued)

\begin{tabular}{|c|c|}
\hline Theme & Illustrative quotes \\
\hline & $\begin{array}{l}\text { "I like the buzz you get and the feeling after that you have accomplished } \\
\text { being able to physically do an activity, everyone in the room... they don't } \\
\text { know I have mild CF and I kind of look and say, god I am able to keep up } \\
\text { with them" (Robert, 41) }\end{array}$ \\
\hline & $\begin{array}{l}\text { "Sometimes it is the best medicine for you, you go off burn some calories, } \\
\text { get a sweat on and you do feel the better for it" (Emily, 50) }\end{array}$ \\
\hline & $\begin{array}{l}\text { "I like the benefits of it, the immediate effects of it and the long term } \\
\text { effects, just keeping the lungs healthy I have to say that's my main benefit } \\
\text { from it"(John, 31) }\end{array}$ \\
\hline & $\begin{array}{l}\text { "The great feeling you have after doing some activity really that you feel } \\
\text { you got loads of energy, you feel like you have actually done something } \\
\text { healthy" (Jason, 35) }\end{array}$ \\
\hline & $\begin{array}{l}\text { "Makes you feel good, even if you are stressed out at home or you had a } \\
\text { bad day... just relieving that stress you have, even if you are tired and you } \\
\text { think ah can't be bothered... you always feel better after it and you say } \\
\text { you are kind of glad [you] went" (Robert, 41); }\end{array}$ \\
\hline
\end{tabular}

perceived competence in their ability to perform PA: "I've been thinking a lot about this, and it's not being able to do it. The thing that turns me off PA is the fear of not being able to do the activity if that makes sense" (Hannah, 36).

\section{Valued outcomes}

Many participants who perceived that they were regularly physically active reported exercise-related outcomes, such as accomplishment and affect regulation as drivers for continued engagement in PA. Many of the participants identified with the importance of keeping healthy: "I mean, just being healthy and not getting sick. Feeling good... and my lung functions being good, that motivates me" (Brenda, 35); "To try and stay as healthy as possible is a motivator" (Richard, 49); "I just realise that if I want to be healthy and if I want to live a kind of normal life, I have to do this...so that pushes me to keep myself as healthy as I can be" (Robert, 41).

Several participants were reportedly driven by a sense of accomplishment: "I do like it in the end...when you feel like you have accomplished something" (Mary, 30); "I like...the feeling after that you have accomplished being able to physically do an activity" (Emily, 50). Affect regulation emerged as a key influencing factor among participants who regularly engaged in PA: "I like how it makes me feel good and I'm always in a better mood, like it releases endorphins and I'm a happier person afterwards definitely" (Brenda, 35); "Just how you feel after doing them, you just feel well and it's good for everything, not just CF, but your mental health and everything... whether you are sick or not, you do feel great about yourself" (Paul, 30).

\section{Preferences}

Many participants reported a preference for exercising within their home environment, as opposed to the gym: "I love doing my treadmill and stuff at home...it reduces the risk of infection as well" (Mary, 30); "At home, just because it's less time consuming and less daunting... it's easier than going to the gym...if your treadmill is sitting there looking at you" (Dorothy, 29); "Home-based...like it can be stressful for me to go to the gym...it's germy and noisy and if you don't feel well you kind of just want to leave once you get there" (Carol, 36).

The concept of attending a gym was perceived negatively by a large number of participants, due to the risk of cross-infection: "They say the worst place to pick up germs that are bad bacteria is in the gym, because everyone is in there sweating. And people that feel sick still go to the gym and they are coughing all over the place and sweating and that kind of puts me off" (Robert, 41); "Going to anything like a gym I just have total war against...I think they're a hive of infection. I think they're the most unclean environments you could possibly get", and illness-related embarrassment: "I don't like when people listen to me coughing when I'm in the gym.... and if I have any secretions, at least if I'm at home, I'll get rid of it there" (Mary, 30); "Going to the gym, especially on Oxygen and things is quite intimidating for people... it's very easy to feel self-conscious so, I think home programmes for people... might help" (William, 34).

Many participants highlighted the Exercise Grant, made available biannually by CF Ireland, as a beneficial tool for enabling home-based exercise: "I have a treadmill at home that I was able to get through the CF Ireland Exercise Grant, which is fantastic, and that is what I would use at home. And, I have got a few little weights... 
it's easier for me than driving to the gym, which takes more time out of my day" (Emily, 50), and "From a CF Ireland point of view, the Exercise Grant is really good because it can kind of help with the financial burden of exercise" (William, 34).

\section{Discussion and conclusion}

\section{Discussion}

The present study is one of the first to explore factors influencing PA in adults with CF. The main barriers among participants were internal (low energy), external (time and the weather) and related to exercise confidence (self-consciousness and capability). The findings of the current study are consistent with previous research which found that the lack of energy and time, and bad weather contributed to poor PA compliance among adults with CF $[5,17]$ and COPD [30]. Exercise-related confidence as a barrier to sustained PA engagement among adults with $\mathrm{CF}$ is a novel finding.

Motivation was a dominant theme underpinned by enjoyment and perceived competence, and appeared to differentiate between those that self-reported being more and less physically active. Specifically, those who reported positive past experiences with $\mathrm{PA}$, higher levels of perceived competence, and more autonomous forms of motivation appeared to engage in more frequent and sustained PA. In contrast, those who reported negative past experiences with PA and lower perceived competence appeared to be less physically active, linked to feelings of external or introjected regulation. Our findings are consistent with the self-determination theory (SDT) in relation to the innate psychological needs (specifically competence and autonomy) that must be satisfied in order to be autonomously and more intrinsically motivated [22]. According to SDT, controlled motivation is when individuals are motivated in order to satisfy some form of external pressure (e.g., physician) or internal pressure (e.g., sense of guilt). Some participants in the current study that were notably less motivated to exercise reported motives driven by extrinsic factors and reported participating in PA to avoid feelings of guilt (introjected regulation). More autonomous motivation is achieved when the individual values health or physical fitness, or when the behaviour (PA) is consistent with his or her ambitions in life or identity (identifying with valued outcomes). Indeed, the present study found that valued exercise-related outcomes were drivers for continued exercise participation amongst participants who appeared to be more regularly physically active. Consistent with SDT and previous research, more autonomous motivation rather than controlled or externally driven motivation are associated with regular exercise behaviour [20, 21]. The strongest predictor of exercise maintenance is personally valued outcomes [31]. Participants in the present study who self-reported to be more regularly active reported affect regulation as a motive for continued engagement in PA. The role of exercise for affect regulation is a novel finding.

A key finding was that most participants, particularly those who were more motivated, expressed a preference for home-based, rather than gym- or facility-based PA. This is notable, since home-based interventions have the capacity to potentially ameliorate feelings of low perceived competence, self-consciousness and remove barriers associated with time and changing weather [22]. It is possible, also that the wider CF population, likely to be less interested in exercise, are unlikely to prefer the gym. It should be acknowledged that the present sample was of an older age profile than the wider Irish CF population, and this may have influenced exercise preferences. Younger CF patients may prefer facility-based exercise, and further research is required to further elucidate exercise preferences in the broader CF population. Tailoring PA programmes according to patient preferences and psychographic profiling [28] may optimise uptake and adherence.

\section{Practice implications}

The reported barriers to PA may be modifiable. For example, self-consciousness and low perceived competence could be mitigated through the provision of mastery experiences to provide a sense of accomplishment and competence. Clinicians could be trained in assisting patients with effective goal-setting, action planning and problem solving for physical activity to provide a sense of accomplishment and alleviate barriers. Homebased interventions that focus on PA rather than facility-based exercise or sport may help alleviate feelings of self-consciousness and low energy. Clinicians could also be trained to help foster autonomous motivation by asking patients to describe some personally valuable outcomes of exercise participation, in addition to providing a rationale for the health benefits of exercise to $\mathrm{CF}$ patients.

Several systematic and meta-analyses have highlighted motivational interviewing (MI) as an approach that has been shown to be effective in changing health behaviours [32]. Providing healthcare professionals with additional training in the use of activity counseling, brief motivational interviewing and techniques to foster the increased importance of PA and confidence to exercise (for example, the use of importance and confidence rulers in brief MI), may have the potential to increase autonomous motivation, and decrease external regulation, toward long-term engagement in PA among patients with $\mathrm{CF}$ [33] and has been recommended in other patient cohorts. 
Motivational interviewing is advantageous in the clinical setting because it is an individualized intervention appropriate to all patients along the continuum of motivation. For those CF patients that are less motivated to change, strategies can focus on raising the importance of physical activity and exploring confidence to change. For those patients more 'ready' to change, more action-oriented strategies such as goal-setting and action planning can be implemented [31]. Individual barriers and exercise preferences can also be explored to devise a patient-centered intervention.

Given the findings in relation to participants' motives and exercise preferences, home-based interventions may hold promise in addition to overcoming the barriers associated with facility-based exercise programmes, such as the fear of exercising in public and the risk of crossinfection. Interventions aimed at developing feelings of competence and a sense of accomplishment (via goal setting) may facilitate increased adherence as they have been found to underpin autonomous motivation. Prior to the development of exercise interventions in this group, further research is needed to confirm or expand the exploratory findings of the present study. Future research that ascertains exercise perspectives of those CF patients less interested in physical activity would be worthwhile. In addition, it would be worthwhile to canvass attitudes towards physical activity engagement amongst CF patients experiencing symptom exacerbations and/or more severe disease progression.

\section{Study limitations}

The study was limited by the relatively low uptake which may not be representative of the wider CF population in Ireland. The absence of exclusion criteria and discussion surrounding the presence of symptoms must also be noted as a limiting factor and the study did not explore whether other comorbidities influenced the results. A further limitation is the absence of objectively-derived PA data for the sample. Opportunistic sampling may have resulted in the recruitment of a more physically active that recognise the benefits of regular PA participation, overlooking the perceptions of those who are disinterested in PA. Although the sample appeared to be similar to the Irish CF population in relation to gender and BMI, lung function was higher in the present study which may indicate that we have recruited participants that are healthier and may have more positive attitudes towards PA. The sample was also older, with a higher proportion having had a transplant which could partially account for the preferences for home-based PA. The findings in the current study are tentative, and it is unclear as to whether they are transferable to the wider CF population. Further research is required to comprehensively evaluate the factors influencing PA among adults with CF. It is also important to acknowledge the relevance of our findings in relation to the Covid-19, and post-Covid-19, environment, highlighting the value of home-based exercise for vulnerable populations, such as those with $\mathrm{CF}$.

\section{Conclusion}

Barriers to PA engagement in the current study were low energy levels, time, the weather, and exercise-related confidence. A tentative but important finding was the preference for home-based rather than gym- or facility-based PA. Effective interventions will likely require the promotion of autonomous motivation, enjoyable activities, personally identified outcomes and competence among adults with CF.

Abbreviations

CF: cystic fibrosis; PA: physical activity.

\section{Supplementary information}

The online version contains supplementary material available at https://doi. org/10.1186/s12890-021-01482-x.

Additional file 1. An interview guide was developed based on previously published work.

\section{Acknowledgements}

We wish to thank each of the participants for volunteering their time to participate in the study, and Cystic Fibrosis Ireland for their assistance in recruitment. We wish give sincere thanks for Cystic Fibrosis Ireland and the Mater Foundation for funding this research.

\section{Authors' contributions}

$\mathrm{NH}$ conceptualization, methodology, investigation, formal analysis, writingoriginal draft. NMM: supervision, conceptualization, writing-review and editing. BK: supervision, conceptualization, writing-review and editing. NM: funding acquisition, writing-review and editing. KR: funding acquisition, writing-review and editing. SH: supervision, formal analysis, writing-original draft, writing-review and editing. All authors read and approved the final manuscript.

\section{Funding}

This research was funded by Cystic Fibrosis Ireland and the Mater Foundation, who provide stipend payment for the lead author.

Availability of data and materials

Data will be made available upon reasonable request.

\section{Declarations}

Ethics Approval and Consent to Participate:

Ethical approval was granted by the Dublin City University Research Ethics Committee (DCUREC/2018/141). I confirm all patient/personal identifiers have been removed or disguised so the patient/person(s) described are not identifiable and cannot be identified through the details of the story. Prior to participation, participants signed and returned an informed consent form, in accordance with the Declaration of Helsinki. The study protocol was also carried out in accordance with the Declaration of Helsinki. 


\section{Consent for Publication \\ Not applicable.}

\section{Competing interests}

The authors do not have any competing interests to declare. The authors have full control of all primary data and agree to allow the Journal to review the data if required.

\section{Author details \\ ${ }^{1}$ School of Health and Human Performance, Faculty of Science and Health, Hospital, Dublin, Ireland. ${ }^{5}$ Institute for Health Research, University of Notre Dame Australia, Fremantle, Australia. \\ Received: 20 November 2020 Accepted: 26 March 2021 Published online: 02 April 2021} Dublin City University, Dublin 9, Ireland. ${ }^{2}$ Department of Sport and Exercise Science, Waterford Institute of Technology, Waterford, Ireland. ${ }^{3}$ ExWell, Chronic Illness Rehabilitation Centre, Dublin, Ireland. ${ }^{4}$ Mater Misericordiae University

\section{References}

1. Swisher AK, Hebestreit H, Mejia-Downs A, Lowman JD, Gruber W, Nippins $M$, et al. Exercise and habitual physical activity for people with cystic fibrosis: expert consensus, evidence-based guide for advising patients. Cardiopulm Phys Ther J. 2015;26(4):85-98.

2. Cox NS, Alison JA, Holland AE. Interventions for promoting physical activity in people with cystic fibrosis. In: Cochrane Cystic Fibrosis and Genetic Disorders Group (eds). Cochrane Database Syst Rev. https://doi.org/10. 1002/14651858.CD009448.pub2

3. Shei R-J, Mackintosh KA, Peabody Lever JE, McNarry MA, Krick S. Exercise physiology across the lifespan in cystic fibrosis. Front Physiol. 2019. Available from: https://www.ncbi.n/m.nih.gov/pmc/articles/PMC6856653/

4. Happ MB, Hoffman LA, DiVirgilio D, Higgins LW, Orenstein DM. Parent and child perceptions of a self-regulated, home-based exercise program for children with cystic fibrosis. Nurs Res. 2013;62(5):305-14.

5. Burnett DM, Barry AN, Mermis JD. Physical activity level and perception of exercise in cystic fibrosis. Respir Care. 2020;65(4):500-6.

6. Williams CA, Stevens D. Physical activity and exercise training in young people with cystic fibrosis: current recommendations and evidence. J Sport Health Sci. 2013;2(1):39-46.

7. Nixon PA, Orenstein DM, Kelsey SF, Doershuk CF. The prognostic value of exercise testing in patients with cystic fibrosis. N Engl J Med. 1992;327(25):1785-8.

8. Vendrusculo FM, Heinzmann-Filho JP, da Silva JS, Perez Ruiz M, Donadio MVF. Peak oxygen uptake and mortality in cystic fibrosis: systematic review and meta-analysis. Respir Care. 2019;64(1):91-8.

9. Hebestreit $\mathrm{H}$. Physical activity is independently related to aerobic capacity in cystic fibrosis. Eur Respir J. 2006;28(4):734-9.

10. Savi D, Di Paolo M, Simmonds N, Onorati P, Internullo M, Quattrucci S, et al. Relationship between daily physical activity and aerobic fitness in adults with cystic fibrosis. BMC Pulm Med. 2015;15(1):59.

11. Cerny F. Exercise and cystic fibrosis (CF) 2.0. Pediatr Exerc Sci. 2013;25(4):616-23.

12. Dwyer TJ, Alison JA, McKeough ZJ, Daviskas E, Bye PTP. Effects of exercise on respiratory flow and sputum properties in patients with cystic fibrosis. Chest. 2011;139(4):870-7.

13. Schneiderman-Walker J, Pollock SL, Corey M, Wilkes DD, Canny GJ, Pedder $L$, et al. A randomized controlled trial of a 3-year home exercise program in cystic fibrosis. J Pediatr. 2000;136(3):304-10.

14. Physical exercise training for cystic fibrosis - Radtke, T (2017) Cochrane Library. https://doi.org/10.1002/14651858.CD002768.pub4/full

15. Hebestreit $H$, Kriemler $S$, Radtke T. Exercise for all cystic fibrosis patients: Is the evidence strengthening? Curr Opin Pulm Med. 2015;21(6):591-5.
16. Swisher PTAK, Pt ME. Perceptions of physical activity in a group of adolescents with cystic fibrosis. Cardiopulm Phys Ther J. 2008;19(4):107-14.

17. Dwyer TJ, Griffin BN, Bye PTP, Alison JA. Elicitation interview study to identify salient beliefs about exercise participation in adults with cystic fibrosis. Physiotherapy. 2020;107:286-91.

18. White D, Stiller K, Haensel N. Adherence of adult cystic fibrosis patients with airway clearance and exercise regimens. J Cyst Fibros Off J Eur Cyst Fibros Soc. 2007;6(3):163-70.

19. Myers LB. An exploratory study investigating factors associated with adherence to chest physiotherapy and exercise in adults with cystic fibrosis. J Cyst Fibros. 2009;8(6):425-7.

20. Ajzen I. The theory of planned behavior. Organ Behav Hum Decis Process. 1991;50:179-211.

21. Ayotte BJ, Margrett JA, Hicks-Patrick J. Physical activity in middle-aged and young-old adults: the roles of self-efficacy, barriers, outcome expectancies, self-regulatory behaviors and social support. J Health Psychol. 2010;15(2):173-85.

22. Ryan RM, Deci EL. Self-determination theory and the facilitation of intrinsic motivation, social development, and well-being. Am Psychol. 2000;11.

23. Maxwell-Smith C, Zeps N, Hagger MS, Platell C, Hardcastle SJ. Barriers to physical activity participation in colorectal cancer survivors at high risk of cardiovascular disease. Psychooncology. 2017;26(6):808-14.

24. Clark JP. How to peer review a qualitative manuscript. In: Godlee F, Jefferson T, editors. Health Sciences. 2nd ed. London: BMJ Books; 2003. p. 219-35.

25. Cantwell M, Walsh D, Furlong B, Loughney L, McCaffrey N, Moyna N, et al. Physical activity across the cancer journey: experiences and recommendations from people living with and beyond cancer. Phys Ther. 2020;100(3):575-85.

26. Braun V, Clarke V. Using thematic analysis in psychology. Qual Res Psychol. 2006;3(2):77-101.

27. Qualitative Research Methods in Sport, Exercise and Health : From Process to Product [Internet]. Routledge; 2013 [cited 2021 Feb 10]. Available from: https://www.taylorfrancis.com/books/qualitative-research-methods-sport-exercise-health-andrew-sparkes-brett-smith/https://doi.org/10. 4324/9780203852187

28. Hardcastle SJ, Hagger MS. psychographic profiling for effective health behavior change interventions. Front Psychol [Internet]. 2016 Jan 6 [cited 2020 Oct 15];6. Available from: https://www.ncbi.nlm.nih.gov/pmc/artic les/PMC4701903/

29. Sandelowski M. Whatever happened to qualitative description? Res Nurs Health. 2000;23(4):334-40.

30. Kosteli M-C, Heneghan NR, Roskell C, Williams SE, Adab P, Dickens $A P$, et al. Barriers and enablers of physical activity engagement for patients with COPD in primary care. Int J Chron Obstruct Pulmon Dis. 2017;12:1019-31.

31. Teixeira PJ, Carraça EV, Markland D, Silva MN, Ryan RM. Exercise, physical activity, and self-determination theory: a systematic review. Int J Behav Nutr Phys Act. 2012;9:78.

32. Hardcastle SJ, Fortier M, Blake N, Hagger MS. Identifying content-based and relational techniques to change behaviour in motivational interviewing. Health Psychol Rev. 2017;11(1):1-16.

33. Denford S, van Beurden S, O'Halloran P, Williams CA. Barriers and facilitators to physical activity among children, adolescents, and young adults with cystic fibrosis: a systematic review and thematic synthesis of qualitative research. BMJ Open [Internet]. 2020 Feb 20 [cited 2020 Oct 15]; 10(2). Available from: https://www.ncbi.nlm.nih.gov/pmc/articles/PMC70 45130/

\section{Publisher's Note}

Springer Nature remains neutral with regard to jurisdictional claims in published maps and institutional affiliations. 